\title{
Estudio descriptivo de la analgesia obtenida durante el trabajo de parto con PCA de remifentanilo: modelo británico
}

\author{
C. Morales Muñoz y A. Albadalejo Serrano ${ }^{2}$ \\ Servicio de Anestesiología. ${ }^{1}$ Hospital Juan Ramón Jiménez, Huelva. ${ }^{2}$ Hospital Rafael Méndez. Lorca, Murcia.
}

Morales Muñoz C, Albadalejo Serrano A. Estudio descriptivo de la analgesia obtenida durante el trabajo de parto con PCA de remifentanilo: modelo británico. Rev Soc Esp Dolor 2014; 21(6): 307-314.

\begin{abstract}
Background: Epidural analgesia in Obstetrics is nowadays the gold standard for pain relief in labour, but in some cases is contraindicated or the analgesia is incomplete. Remifentanil is a strong, ultra-short-acting and fast onset opioid that timing very well with the dynamic of the labour. The coordination between the beginning of the uterus contraction and the drug administration with the PCA device makes this technique could be considered as election for pain relieve in labour and not only as an alternative to epidural.

Objective: This study aims to assess the efficacy and safety of the intravenous remifentanil in labour and the maternal satisfaction.

Materials and methods: Descriptive and prospective study based on the review of the dates recorded by the investigators during the labour where it's registered the pain along the dilatation and the expulsive before and after applied the remifentanil PCA, and the complications and the incidents of secondary effects as well. At the same time it's made an inquiry about maternal satisfaction about the technique received and their personal experience.

Results: 32 cases have been recorded in total during the study period (6 months). All patients have been presented a reduction of the pain displayed by a reduction of the EVA in 5.9 points in the first minutes, 4.6 in completed, and 3.4 in the expulsive. The maternal satisfaction has been high, and $90 \%$ of parturient would be repeated the technique.
\end{abstract}

Conclusions: Remifentanil PCA has been seemed effective and safe as analgesic in Obstetrics. It's for that remifentanil PCA has been had a great acceptation within midwifes, gynaecologist and anaesthetist in our hospital, currently it is been offered as first option as epidural, depending on the patient, obstetrician and anaesthetist opinion.

Key words: Remifentanil. Analgesia. Labour. PCA.

\section{RESUMEN}

Introducción: la analgesia epidural obstétrica continúa siendo el "gold-standard" para controlar el dolor durante el trabajo de parto, pero en determinadas ocasiones la técnica está contraindicada o la analgesia es incompleta. El remifentanilo es un opioide potente de rápida acción y vida media ultracorta, que se adapta perfectamente a la dinámica del parto. La coordinación entre el comienzo de las contracciones y la administración del fármaco mediante un dispositivo PCA hacen que esta técnica pueda ser considerada de elección para el alivio de dolor durante el parto y no solo como alternativa a la epidural.

Objetivo: este estudio pretende valorar la eficacia y seguridad en el parto del remifentanilo administrado por vía intravenosa, así como la satisfacción materna de la analgesia recibida.

Materiales y métodos: estudio descriptivo prospectivo basado en el análisis de la recogida de datos realizado por los investigadores durante el parto donde se recoge el dolor durante la dilatación y el expulsivo antes y después de la aplicación de la PCA de remifentanilo, así como las complicaciones y la incidencia de efectos secundarios. Paralelamente se realiza una encuesta de satisfacción materna de la técnica recibida y su vivencia personal.

Resultados: se han recogido un total de 32 casos durante el periodo de estudio (6 meses). Todas las pacientes han presentado una reducción de dolor manifestado por una disminución del EVA respecto del dolor basal de 5,9 puntos en los primeros minutos, 4,6 puntos en completa y 3,4 en el expulsivo. No se han registrado complicaciones materno-fetales importantes derivadas de la técnica. La satisfacción materna ha sido alta, volviendo incluso a repetir la técnica en un $90 \%$ de los casos. 
Conclusiones: la PCA de remifentanilo ha demostrado ser efectiva y segura como analgesia obstétrica. Es por ello que la PCA ha teniendo una amplia aceptación entre matronas, ginecólogos y anestesiólogos en nuestro hospital, siendo ofrecida actualmente como primera opción junto con la epidural a criterio de elección de la paciente, obstetra y anestesiólogo.

Palabras clave: Remifentanilo. Analgesia. Parto. PCA.

\section{INTRODUCCIÓN}

La epidural continúa siendo en España el método de elección para controlar el dolor durante el parto. Se estima que los médicos españoles presentaron servicios anestésicos para el parto (vaginal y cesárea) en el $82 \%$ de los casos (1) en el año 2006. Posiblemente la tasa más alta del mundo de las registradas en la bibliografía hasta el momento. La incidencia de la epidural para el parto en nuestro país se ha incrementado considerablemente en la última década, se estima en torno al 83-98\% según los diversos estudios revisados $(1,2)$. No existe duda de su indicación, eficacia y seguridad en obstetricia, pero el retraso en la edad gestacional implica un paulatino envejecimiento de la población obstétrica, con mayor comorbilidad asociada, donde la epidural podría estar incluso contraindicada (3). Este hecho, junto con el aumento de la obesidad en países del primer mundo como es el caso de España, hacen que la técnica sea más complicada, y por lo tanto aumenten los riesgos de punción dural, analgesia incompleta o fallo de la técnica. Es por ello que técnicas alternativas como la PCA (patient control analgesia) de remifentanilo hayan ido ganando importancia en los últimos años en países como Inglaterra, ya que ha demostrado resultar efectiva y segura para el control del dolor producido por las contracciones durante el trabajo de parto y el expulsivo, presentando una similar incidencia en su uso en la actualidad en nuestro hospital (21\%, frente a un $24 \%$ en las epidurales).

Se estima que más del $50 \%$ de las inglesas (4) prefieren no usar la epidural durante el parto por miedo, desconfianza o uso de otras técnicas alternativas menos efectivas que la epidural, como el óxido nitroso (Entonox ${ }^{\circledR}$ ), TENS (neuroestimulador transcutáneo), petidina intramuscular, inmersión en agua, aromaterapia o pilates. Ante esta nueva situación social donde la analgesia epidural puede ser dificultosa e incluso estar contraindicada, se decidió implantar en nuestro hospital un nuevo sistema de analgesia para el dolor de parto: la PCA de remifentanilo.

El remifentanilo (4-anilido-piperidine) es un opioide sintético que proporciona analgesia rápida y potente $(5,6)$. Se caracteriza por tener una vida media ultracorta, con un pequeño volumen de distribución $(0,391 / \mathrm{kg} \pm 0,25)$, una rápida fase de redistribución $(0,94 \pm 0,57 \mathrm{~min})$ y una vida media de 9,5 \pm 4 min (8,9-1). El equilibrio entre los compartimentos sangre-cerebro ocurre en 1,2-1,4 min (3). En general, la vida media del fármaco es de 3,5 minutos independientemente de la duración de la infusión. El remifentanilo atraviesa la barrera hematoplacentaria y es metabolizado por las esterasas plasmáticas y tisulares no específicas tanto maternas como fetales.

Por todo lo anterior, la farmacocinética del mismo se adapta perfectamente para aliviar al dolor producido por las contracciones uterinas. Su rápida acción hace que tras administrarlo, la paciente sienta alivio en unos 30-60 segundos, mostrando el pico máximo de analgesia a los 2,5 minutos. La coordinación entre el comienzo de las contracciones y la administración del fármaco mediante un dispositivo PCA (que ofrece mayor precisión y seguridad) hacen que esta técnica pueda ser considerada de elección para el alivio de dolor durante el parto y no sólo como alternativa en aquellos casos donde la epidural esté contraindicada.

\section{OBJETIVO}

El objetivo de nuestro estudio es medir la efectividad, seguridad, así como la satisfacción materna, tras recibir analgesia con PCA de remifentanilo para controlar el dolor durante el trabajo de parto y el expulsivo.

\section{MATERIALES Y MÉTODO}

Para ello hemos diseñado un estudio prospectivo observacional basado en el análisis de la recogida de datos (Fig. 1) realizado por los investigadores (médicos residentes y adjuntos de anestesia y matronas) durante el periodo de dilatación y el expulsivo, de pacientes que han solicitado recibir analgesia con PCA de remifentanilo. A su vez se realizó una encuesta a la paciente tras el parto para indagar acerca de su satisfacción y su experiencia personal (Fig. 2).

Los criterios de inclusión son: pacientes en trabajo de parto que soliciten la PCA de remifentanilo y no cumplan ningún criterio de exclusión (parto múltiple, alergia a morfina o derivados, menos de 36 semanas de gestación, rechazo de la técnica, uso de opiáceo por vía IV o IM en las últimas 4 horas).

Tras explicar a la paciente los riesgos y beneficios de la técnica, y obtener un consentimiento informado, se procede a canalizar una nueva vía periférica calibre $20 \mathrm{G}$ para uso exclusivo de la PCA de remifentanilo.

Según el protocolo del servicio (Tabla I) la PCA es programada por el anestesiólogo encargado de la paciente, por el cual se prepara remifentanilo $40 \mu \mathrm{g} / \mathrm{ml}$ en una jeringa de $50 \mathrm{ml}$. La bomba utilizada es Graseby Omnifuse PCA ${ }^{\circledR}$, programada en modo de PCA pura, bolos de $1 \mathrm{ml}(40 \mu \mathrm{g})$ cada 2 minutos (tiempo de cierre) inyectado en 30 segundos. 


\section{HOJA DE RECOGIDA DE DATOS: PCA DE REMIFENTANILO}

Pegatina identificadora de la paciente: nombre/fecha de nacimiento/número de la seguridad social
Fecha:

Anestesiólogo:

Prescripción: PCA remifentanilo $40 \mu \mathrm{g} / \mathrm{ml}$

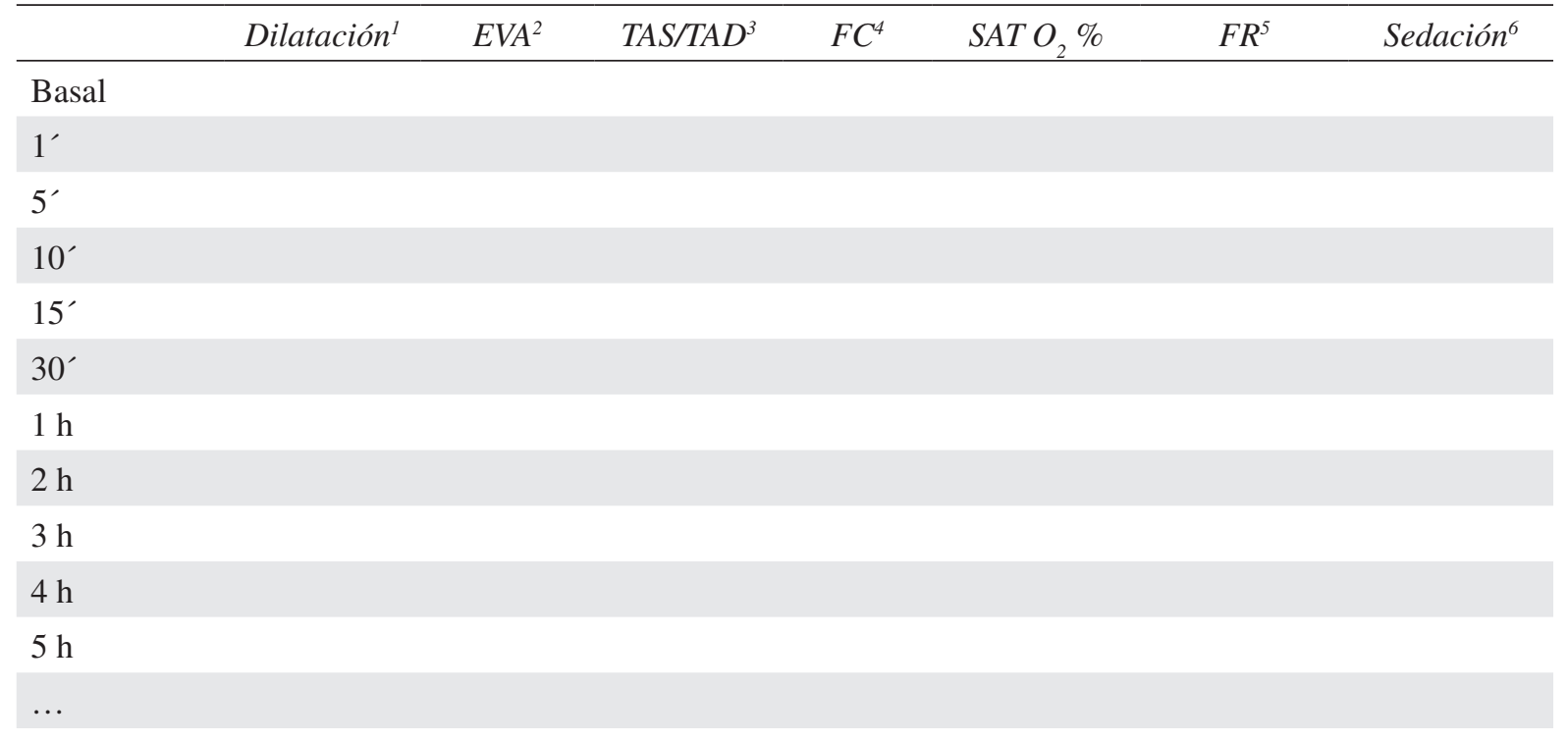

\section{EXPULSIVO}

DOLOR (EVA):

SEDACIÓN:

CAPACIDAD DE EMPUJAR:

( 0 no empuja, 1 empuja con esfuerzo, 2 empuja bien, 3 empuja con fuerza)

COMPLICACIONES:

\section{BEBÉ}

SEXO:

PESO:

TEST DE APGAR (0/10):

1':

5':

10':

COMPLICACIONES:

'Dilatación cervical.

2EVA: escala visual analógica (0-10), donde 10 es el máximo dolor experimentado y 0 ausencia de dolor.

${ }^{3}$ TAS: tensión arterial sistólica, TSD: tensión arterial diastólica

${ }^{4} \mathrm{FC}$ : frecuencia cardiaca.

${ }^{5} \mathrm{FR}$ : frecuencia respiratoria.

${ }^{6}$ Sedación: escala de sedación: 0 Alerta, 1 Ocasionalmente dormido, 2 Dormido de fácil despertar,

3 Dormido de difícil despertar, 4 No responde a la llamada

Fig. 1. Hoja de recogida de datos. 


\section{ENCUESTA DE SATISFACCIÓN: PCA DE REMIFENTANILO}

- Valore del 1 al 10 lo satisfecha que ha estado con la PCA de remifentanilo durante la dilatación para el parto. Siendo 0 nada satisfecha y 10 lo más satisfecha posible:

- Valore del 1 al 10 lo satisfecha que ha estado con la PCA de remifentanilo durante el expulsivo. Siendo 0 nada satisfecha y 10 lo más satisfecha posible:

- ¿Volvería a repetir la PCA de remifentanilo si tuviera otro embarazo?:
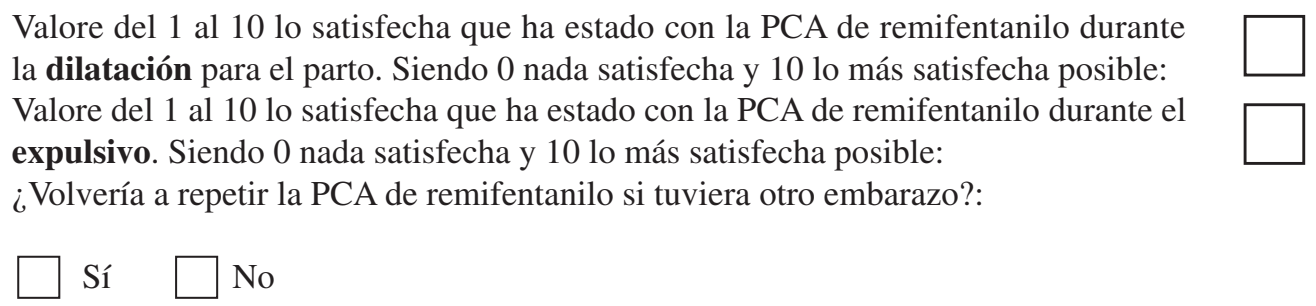

- ¿Le han puesto la epidural anteriormente?, ¿cuál ha satisfecho mejor sus necesidades?

- Lo mejor de la PCA ha sido:

- Lo peor de la PCA ha sido:

COMENTARIOS:

Fig. 2. Encuesta de satisfacción.

\begin{tabular}{|c|c|c|}
\hline \multicolumn{3}{|c|}{ TABLA I. PROTOCOLO DEL SERVICIO } \\
\hline $1^{\circ}$ & $\begin{array}{l}\text { Obtener } \\
\text { consentimiento } \\
\text { informado de la } \\
\text { paciente }\end{array}$ & $\begin{array}{l}\text { Contraindicaciones: gestación } \\
\text { múltiple, uso de opioide en } \\
\text { las } 4 \text { horas previas, alergia/ } \\
\text { intolerancia a morfina o } \\
\text { derivados. Adicción a drogas } \\
\text { por vía parenteral }\end{array}$ \\
\hline $2^{\circ}$ & \multicolumn{2}{|c|}{$\begin{array}{l}\text { Canalizar vía periférica: } 20 \text { G, para uso exclusivo } \\
\text { de la PCA }\end{array}$} \\
\hline $3^{\circ}$ & \multicolumn{2}{|c|}{$\begin{array}{l}\text { Programar PCA de remifentanilo en modo de } \\
\text { PCA pura: bolos de } 40 \mathrm{mg} \text {, tiempo de cierre } \\
2 \text { minutos. Bomba Graseby Omnifuse PCA }\end{array}$} \\
\hline $4^{\circ}$ & $\begin{array}{l}\text { Monitorización } \\
\text { continua } \\
\text { de } \mathrm{SaO}_{2} \text { y } \\
\text { administración } \\
\text { de oxígeno en } \\
\text { gafas nasales a } \\
\text { 3-8 lpm }\end{array}$ & $\begin{array}{l}\text { La matrona encargada de la } \\
\text { paciente permanece en la } \\
\text { habitación mientras la paciente } \\
\text { tenga la PCA y se encarga del } \\
\text { registro cada } 30 \text { minutos de } \\
\text { frecuencia respiratoria, nivel de } \\
\text { sedación y grado de dolor }\end{array}$ \\
\hline
\end{tabular}

Contactar con anestesia si: excesiva sedación,

$5^{\circ}$ frecuencia respiratoria menor de 8 respiraciones por minuto, $\mathrm{SatO}_{2}$ menor de $90 \%$

Se le explica a la paciente la sistemática de la PCA, haciendo hincapié en que debe pulsar el botón nada más sentir que la contracción empieza y nunca entre contracciones. Sólo la paciente puede pulsar el botón de la PCA, ni familiar, ni matrona, ni médico pueden activarla. Siguiendo el protocolo, se prescribe oxígeno en gafas nasales, ondansetrón (para controlar las náuseas o vómitos que pudieran aparecer como efectos secundarios) y naloxona (para tratamiento en caso de parada respiratoria).

Tras conectar la PCA a la paciente y comprobar que la paciente entiende el funcionamiento de la misma y la importancia de la sincronización de la PCA con el comienzo de las contracciones, el anestesiólogo permanece en la habitación durante los primeros 15 minutos tras la analgesia con remifentanilo, observando que la paciente tolera la PCA, no se produce desaturación de oxígeno, parada respiratoria o ninguna otra complicación derivada de la misma.

La matrona es la encargada de monitorizar las constantes de la paciente durante todo el tiempo que dure la infusión de remifentanilo y de rellenar la hoja de recogida de datos, así como de proporcionarle a la paciente la encuesta de satisfacción personal de la PCA de remifentanilo tras el parto.

\section{RESULTADOS}

Se obtienen un total de 32 casos durante los 6 meses del periodo de estudio realizado. Las características de la población obstétrica quedan reflejadas en la tabla II.

En cuanto al grado de dolor durante la dilatación, todas las pacientes presentaron un alivio de dolor importante manifestado por la disminución del EVA tras comenzar con la PCA de remifentanilo (Tabla III) al igual que durante el periodo del expulsivo, donde se produjeron dos casos de intenso dolor debido a la salida accidental de la vía periférica por la que se 
TABLA II. DATOS ANTROPOMÉTRICOS DE LAS PACIENTES ESTUDIADAS

\begin{tabular}{|c|c|}
\hline & Grupo de estudio $(n=32)$ \\
\hline Edad (media años) & $29+/-8$ \\
\hline Peso (media kg) & $82 \pm 11$ \\
\hline Altura (media cm) & $160+/-6$ \\
\hline ASA I-II-III & $\begin{array}{l}\text { ASA I } n=15(46 \%) \\
\text { ASA II } n=13(40 \%) \\
\text { ASA III } n=4(12 \%)\end{array}$ \\
\hline Primípara/multípara & $\mathrm{n}=2165 \% / \mathrm{n}=1134 \%$ \\
\hline Indicación & $\begin{array}{l}\text { Solicitud materna } \mathrm{n}=28(87 \%) \\
\text { Fallo de la epidural } \mathrm{n}=1(3 \%) \\
\text { Analgesia incompleta con la } \\
\text { epidural } \mathrm{n}=3(9 \%)\end{array}$ \\
\hline
\end{tabular}

TABLA III. EVOLUCIÓN DE LA ESCALA VISUAL ANALÓGICA (EVA), DE LA SEDACIÓN Y DEL BLOQUEO MOTOR

\begin{tabular}{lccc}
\hline & $\begin{array}{c}\text { Dolor } \\
(\text { EVA: 0-10) }\end{array}$ & Sedación & $\begin{array}{c}\text { Bloqueo } \\
\text { motor }\end{array}$ \\
\hline Basal & $9,2(7-10)$ & 0 & 0 \\
1 minuto & $5,2(4-9)$ & $0,4(0-1)$ & 0 \\
10 minutos & $3,3(2-5)$ & $1,4(1-2)$ & 0 \\
1 hora & $3,8(2-6)$ & $1,6(1-2)$ & 0 \\
Dilatación completa & $4,6(2-7)$ & $0,6(0-2)$ & 0 \\
Expulsivo & $5,8^{*}(3-8)$ & $0,4(0-1)$ & 0 \\
Parto & $5,7^{*}(4-8)$ & $0,4(0-1)$ & 0 \\
\hline
\end{tabular}

*2 pacientes fueron excluidas por salida accidental de la vía durante el expulsivo y el parto.

administraba la PCA de remifentanilo (sin dar tiempo a recolocarla antes del parto). En general todas las pacientes han presentado una reducción de dolor manifestado por una disminución del EVA con respecto del dolor basal de 5,9 puntos en los primeros minutos, 4,6 en completa y 3,4 en el expulsivo.

Las pacientes no presentaron bloqueo motor en ningún caso, y tuvieron fuerza suficiente para empujar durante el expulsivo; sólo dos casos requirieron parto con fórceps y tres casos acabaron en cesárea. El grado de sedación tampoco se consideró un problema durante el expulsivo.

No se registró ninguna complicación importante derivada de la técnica (Tabla IV). No hubo ningún caso de desaturación o parada respiratoria. El test de Apgar del recién nacido en los primeros minutos se mantuvo dentro de la normalidad en todos los casos (Tabla V).
Analizando la satisfacción materna (Tabla VI), la mayoría de las pacientes quedaron muy satisfechas con la PCA

\section{TABLA IV. COMPLICACIONES}

\begin{tabular}{ll}
\hline Náuseas/vómitos & $\begin{array}{l}2 \text { casos (náuseas previas /uso } \\
\text { Entonox }^{\circledR} \text { ) }\end{array}$ \\
$\begin{array}{l}\text { Depresión respiratoria } \\
\text { Sedación profunda }\end{array}$ & 0 \\
ECG fetal patológico & 1 caso \\
Instrumentación & 2 \\
Cesáreas & 3 casos (fallo progresión, \\
& $\begin{array}{l}\text { 1 sufrimiento fetal) } \\
\text { 2 casos de salida accidental de la } \\
\text { vía con la PCA de remifentanilo } \\
\text { durante el expulsivo }\end{array}$ \\
\hline
\end{tabular}

TABLA V. TEST DE APGAR DEL RECIÉN NACIDO

\begin{tabular}{ll}
\hline Minuto 1 & $9(7-9)$ \\
Minuto 5 & $9(8-10)$ \\
Minuto 10 & $9(9-10)$ \\
\hline
\end{tabular}

TABLA VI. SATISFACCIÓN MATERNA

\begin{tabular}{ll}
\hline $\begin{array}{l}\text { Satisfacción en } \\
\text { dilatación (0-10) }\end{array}$ & 8,4 \\
\hline $\begin{array}{l}\text { Satisfacción } \\
\text { expulsivo (0-10) }\end{array}$ & 7,2 \\
\hline $\begin{array}{l}\text { Repetiría PCA de } \\
\text { remifentanilo }\end{array}$ & $90 \%$ Sí \\
& $\begin{array}{l}\text { 4\% No lo sé } \\
6 \% \text { No }\end{array}$ \\
\hline Lo mejor & - "Alivio instantáneo del dolor" \\
& - "Podía empujar con fuerzas" \\
& - "Podía moverme bien por la cama" \\
& - "No necesité sonda vesical" \\
& - "Se me salió la vía justo antes \\
& del parto y tuve mucho dolor. \\
& $\begin{array}{l}\text { No dio tiempo al anestesista de } \\
\text { ponerme otra vez la PCA" }\end{array}$ \\
& - "Si el anestesista tarda en venir \\
& para reponer la medicación, se \\
& vuelve a tener mucho dolor, peor \\
& que al principio" \\
& - "Al principio me fue muy bien, \\
& pero las últimas horas no fue \\
& suficiente para controlar el dolor" \\
- & "Nada" \\
\hline &
\end{tabular}


de remifentanilo, volviéndola a utilizar como primera opción si tuvieran otro embarazo en un $90 \%$ de los casos.

Es importante destacar que dos casos solicitaron analgesia epidural como primera opción, pero por fallo de la técnica y analgesia incompleta, las pacientes recibieron la PCA de remifentanilo satisfactoriamente. Por otro lado, hubo un caso de analgesia insuficiente con la PCA de remifentilo que requirió conversión a analgesia epidural.

\section{DISCUSIÓN}

La PCA de remifentanilo ha demostrado resultar efectiva para controlar el dolor durante el trabajo de parto y segura tanto para la madre como para el niño, registrándose un EVA por debajo de 4 en las primeras horas y de $4-5$ durante el expulsivo. Estos datos concuerdan con la mayoría de los estudios revisados (7-11) donde la PCA de remifentanilo ha supuesto una reducción moderada en la escala de dolor durante el periodo de dilatación, permaneciendo algo más alto durante el expulsivo.

La eficacia de la PCA de remifentanilo depende tanto de la dosis del fármaco utilizada como de su régimen o pauta de administración. En la actualidad existen numerosos estudios con una amplia variedad de dosis de remifentanilo administrada que varían desde 0,2 hasta $0,93 \mu \mathrm{g} / \mathrm{kg}$ por bolo (9-16). En este estudio hemos establecido un sistema de PCA pura, sin infusión basal, con bolos a demanda de $40 \mu \mathrm{g}$, cada 2 minutos, a pesar de que la mayoría de los estudios obtienen mejores resultados cuando la PCA es administrada según el peso de la paciente. Esta es una de las limitaciones de nuestro estudio, ya que al utilizar una dosis fija, podemos incurrir en una sobredosis (potenciando los efectos secundarios y complicaciones) o una infradosis (obteniendo una analgesia insatisfactoria). Al tratarse de una técnica de reciente incorporación en nuestro hospital, decidimos simplificar la técnica utilizando dosis fijas seguras hasta analizar la seguridad y viabilidad de la técnica en nuestro servicio. Actualmente estamos trabajando en un nuevo protocolo de administración de la PCA de remifentanilo según el peso de la paciente. Otros autores como Evron y cols. (12) proponen un régimen donde van incrementando la dosis del bolo de remifentanilo en $5 \mu \mathrm{g}$ cada 15 minutos hasta obtener una analgesia satisfactoria de la paciente.

Otro aspecto interesante a analizar es la manera de administrar el bolo y el tiempo de cierre de la bomba. Blair y cols. (13) defienden el uso de una PCA pura administrando el bolo de remifentanilo ( $40 \mu \mathrm{g}$ ) en 18 segundos, frente a otros estudios que lo administran en 1 minuto. El tiempo de cierre varía de 1 a 3 minutos en los distintos estudios revisados (12-20), con una amplia mayoría de 2 minutos.

En cuanto al uso de una infusión basal de remifentanilo con bolos a demanda, frente a una PCA pura, no existe una clara tendencia, encontrándose distintos puntos de vista: Blair y cols. (13) afirmaron encontrar mayores efectos secundarios en las pacientes que recibieron la PCA con una infusión basal, sin obtener mejoras en el EVA. Sin embargo, Balki (14) obtiene mejores resultados usando una infusión basal de $0,025-0,1 \mu \mathrm{g} / \mathrm{kg}$ con bolos de $0,25 \mu \mathrm{g} / \mathrm{kg}$ cada 2 minutos que con PCA pura.

Otro punto de controversia en cuanto al régimen de administración de la bomba ha sido cuándo es el mejor momento para activar la PCA de remifentanilo. La mayoría de los estudios consideran que el mejor momento es cuando la paciente sienta que una contracción comienza y no entre las contracciones. Volmanen y cols. (21) vieron que administrando bolos de remifentanilo entre contracciones no mejoraba la analgesia ni disminuían los efectos secundarios.

La principal limitación de los opioides administrados durante el trabajo de parto son sus efectos secundarios como: sedación, hipoventilación, desaturación, náuseas y vómitos (12-22). El remifentanilo, debido a su rápida metabolización por las esterasas plasmáticas y tisulares no específicas tanto maternas como fetales, es un fármaco seguro en obstetricia. Los principales efectos secundarios recogidos en el estudio han sido: náuseas y vómitos $5 \%$ ( 2 casos), sin ningún caso de hipoventilación o desaturación de oxígeno ya que por protocolo administramos oxígenos con gafas nasales a todas la mujeres que reciben PCA de remifentanilo. En los estudios revisados se ha encontrado una amplia variabilidad de efectos secundarios registrados; la incidencia de náuseas y vómitos varía desde $0 \%$ (12)$60 \%$ (14). La mayoría de estos estudios describen algún caso de desaturación que requiere administración de oxígeno (23), siendo interesante destacar el trabajo realizado por Griffin y cols. (24) en el que analizaron las distintas causas de hipoxema materna durante el trabajo de parto y observaron que el grupo que no recibía analgesia registró un mayor número de casos de desaturación que el grupo con epidural, PCA de remifentanilo, meperidina u óxido nitroso. La sedación fue registrada por la mayoría de los estudios como efecto secundario pero nunca considerada como excesiva.

Ningún trabajo ha identificado un aumento en la incidencia de bradicardia fetal o ECG patológico en relación con el uso de la PCA de remifentanilo. El test de Apgar y los gases de la arteria umbilical analizados en los distintos estudios estuvieron dentro de los límites de la normalidad. Ningún niño requirió administración de naloxona en el postparto inmediato. Los datos concuerdan con los resultados obtenidos en nuestro estudio, donde sólo se registró un caso de bradicardia fetal, antes y durante la PCA de remifentanilo, que requirió cesárea por confirmación de nudo de cordón.

Otra limitación de nuestro estudio ha sido el uso concomitante de óxido nitroso $\left(\mathrm{N}_{2} 0\right)$ durante el trabajo de 
parto en la mayoría de las pacientes estudiadas. El Entonox $^{\circledR}$ (mezcla de óxido nitroso/oxígeno al $50 \%$ ) es un gas ampliamente utilizado en Reino Unido; se estima que el $60 \%$ de las inglesas lo usan en algún momento durante el parto (25). El $\mathrm{N}_{2} 0$ es un gas analgésico y ansiolítico de potencia limitada a baja dosis, se absorbe por los pulmones y sus efectos analgésicos son debidos a un aumento en la sangre de endorfinas, cortisol y dopamina. Actualmente se usa en países como Escandinavia, Inglaterra, Canadá, Suecia, Finlandia o Australia como analgésico-ansiolítico durante el parto (26). Su uso se ve limitado por sus efectos secundarios: náuseas y vómitos, mareo, somnolencia o amnesia, así como por proporcionar una analgesia insuficiente para el parto. Se han descrito casos de depresión de la médula ósea, neuropatía y problemas de fertilidad, en exposiciones prolongadas al mismo (27). $\mathrm{El}_{2} 0$ se elimina a la atmósfera contribuyendo al efecto invernadero del planeta. A pesar de que el gas fue solicitado y utilizado por el $75 \%$ de las pacientes antes de comenzar con la analgesia de remifentanilo, el EVA basal fue similar en todas las mujeres del estudio, con lo que consideramos que la reducción en el EVA fue debido al remifentanilo y no al $\mathrm{N}_{2} 0$, aunque consideramos que sí podría estar relacionado con el principal efecto secundario registrado: náuseas y vómitos.

La satisfacción materna tras la experiencia con la PCA de remifentanilo ha sido alta (8,4/10 en dilatación y 7,2/10 en el expulsivo), volviéndola a utilizar como primera opción en un futuro embarazo en el $90 \%$ de los casos. Este hecho concuerda con los estudios revisados $(18,19,28)$ donde la satisfacción materna en pacientes con epidural y pacientes con PCA de remifentanilo eran comparables, a pesar que la reducción de dolor era mayor en el grupo con epidural. Esto puede ser debido a que la satisfacción materna también se ve influenciada por factores personales como: las expectativas previas de la paciente, vivencia anterior, relación con el personal (matrona, obstetra, anestesiólogo), calidad de los cuidados e información recibida, nivel socio-cultural, etc.

Podemos concluir nuestro estudio afirmando que la PCA de remifentanilo ha resultado ser efectiva para controlar el dolor durante el trabajo de parto con una potencia moderada. Se necesitan más estudios prospectivos para establecer el régimen óptimo de administración de la PCA para que resulte más efectiva y segura: dosis $/ \mathrm{kg}$, tiempo de cierre, uso de una infusión basal con o sin bolos a demanda o una PCA pura, momento de administrar el bolo (antes o después de las contracciones), etc. La PCA debe ser supervisada en todo momento por un médico anestesiólogo, la matrona encargada de la paciente debe permanecer en la habitación todo el tiempo que dure la infusión, monitorizando la $\mathrm{SaO}_{2}$ y registrando cualquier complicación que pudiera resultar de la misma.

La PCA ha teniendo una amplia aceptación entre matronas, ginecólogos y anestesiólogos en nuestro hospital, sien- do ofrecida actualmente como primera opción junto con la epidural a criterio de elección de la paciente/obstetra/ anestesiólogo.

\author{
CORRESPONDENCIA: \\ Clara Morales Muñoz \\ Servicio de Anestesiología \\ Hospital Juan Ramón Jiménez \\ Ronda Exterior Norte $\mathrm{s} / \mathrm{n}$ \\ 21005 Huelva \\ e-mail: morales.munoz.clara@gmail.com
}

\section{BIBLIOGRAFÍA}

1. Sabaté S, Gomar C, Canet J, Fernández C, Fernández M. Anestesia obstétrica en Cataluña. Med Clin (Barc) 2006;126(Supl. 2):40-5.

2. Fernández-Guisasola J, Rodríguez Caravaca G, Serrano Rodríguez ML, Delgado González T, García del Valle S, González-Arnau JI. Analgesia epidural obstétrica: relación con diversas variables obstétricas y con la evolución del parto. Rev Esp Anestesiol Reanim 2004;51:121-7.

3. Schearz GL, Volmanen P, Albrechtsen S, Bjoernestad E. Remifentanil target-controlled infusion during second stage labour in high-risk parturients: A case series. Acta Anaesthesiol Scand 2013 Mar 15:doi 10.111.

4. Blair JM, Hill DA, Fee JPH. Patient-controlled analgesia for labour using remifentanil: A feasibility study. Br J Anaesth 2001;87(3):415-20.

5. Hinova A, Fernando R. Systemic remifentanil in labor analgesia. Anesth Analg 2009;109:1925-9.

6. López-Millan JM, et al. Analgesia del trabajo de parto con remifentanilo por vía intravenosa mediante un sistema de analgesia controlada por la paciente PCIA. Rev Soc Esp del Dolor 2007;6:416-21.

7. Schnabel A, Hahn N, Broscheit J, Muellenbach RM, Rieger L, Roewer N, et al. Remifentanil for labour analgesia: a meta-analysis of randomised controlled trials. Eur J Anaesthesiol 2012;29(4):177-85.

8. Anim-Somuah M, Smyth RM, Jones L. Epidural versus non-epidural or no analgesia in labour. Cochrane Database Syst Rev 2011;(12).

9. Jost A, Ban B, Kamenik M. Modified patient-controlled remifentanil bolus delivery regimen for labour pain. Anaesthesia 2013, 68, 245-252.

10. Jones L, Othman M, Dowswell T, Alfirevic Z, Gates S, Newburn M, et al. Pain management for women in labour: an overview of systematic reviews. Cochrane Database Syst Rev 2012;3.

11. Leong WL, Sng BL, Sia AT. A comparison between remifentanil and meperidine for labor analgesia: A systematic review. Anesth Analg 2011;113:818-25.

12. Evron S, Glezerman M, Sadan O, Boaz M, Ezri T. Remifentanil: A novel systemic analgesic for labour pain. Anaesth Analg 2005;100:233-8.

13. Blair JM, Hill DA, Free JP. Patient controlled analgesia for labour: A comparison of remifentanil with pethidine. Anaesthesia 2005;60:22-7.

14. Balki M, Kasodekar S, Dhumne S, Bernstein P, Carvalho JC. Remifentanil patient-controlled analgesia for 
labour: Optimizing drug delivery regimens. Can J Anaesth 2007;54:626-33.

15. Volmanen P, Akural EI, Raudaskoski T, Alahuhta S. Remifentanil in obstetric analgesia: A dose-finding study. Anesth Analg 2002;94:913-7.

16. Volikas I, Butwick A, Wilkinson C, Pleming A, Nicholson G. Maternal and neonatal side-effects of remifentanil Patient-controlled analgesia: a dose-finding study. Anesth Analg 2002;94:913-7.

17. Calderón E, et al. Remifentanilo intravenoso mediante infusor elastomérico frente a meperidina intramuscular. Estudio comparativo en analgesia obstétrica. Rev Soc Esp Dolor 2006;7:462-7.

18. Volmanen P, Sarvela J, Akural EI, Raudaskoski T, Korttila K, Alahuhta S. Intravenous remifentanil vs. Epidural levobupivacaine with fentanyl for pain relief in early labour: a randomised, controlled, double-blinded study. Acta Anaesthesiol Scand 2008;52(2):249-55.

19. Douma MR, Middeldorp JM, Verwey RA, Dahan A, Stienstra R. A randomised comparison of intravenous remifentanil patient-controlled analgesia with epidural ropicavaine/ sulfentanil during labour. Int J of Obste Anesth 2011;20:11823.

20. Douma MR, Verwey RA, Kam-Endtz CE, van der Linden PD, Stienstra R. Obstetric analgesia: A comparison of pateient-controlled meperidine, remifentanil, and fentanyl in labour. Br J Anaesth 2010;104:209-215.
21. Volmanen PV, Akural EI, Raudaskoski T, Ranta P, Tekay A, Ohtonen P, et al. Timing of intravenous patient-controlled remifentanil bolus during early labour. Acta Anaesthesiol Scand 2011;55:486-94.

22. Shen MK, Wu ZF, Zhu AB, He LL, Shen XF, Yang JJ, et al. Remifentanil for labour: A double blinded, randomised controlled trial of maternal and neonatal effects of patient-controlled analgesia versus continuous infusion. Anaesthesia 2013;68:236-44.

23. Bonner JC, McClymont W. Respiratory arrest in an obstetric patient using remifentanil patient-controlled analgesia. Anaesthesia 2012;67:538-40.

24. Griffin RP, Reynolds F, Maternal hypoxemia during labour and delivery: The influence of analgesia and the effect on neonatal outcome. Anaesthesia 1995;50:151-6.

25. Volmanen P, Palomäki O, Ahonen J. Alternatives to analgesia for labor. Curr Opin Anesthesiol 2011;24:235-41.

26. Rooks JP. Labour pain management other tan neuraxial: What do we know and where do we go next? Birth 2012;39(4):318-22.

27. Klomp T, van Poppel M, Jones L, Lazet J, Di Nisio M, Lagro-Janssen AL. Inhaled analgesia for pain management in labour. Cochrane Database Syst Rev 2012;9.

28. Tveit TO, Seiler S, Halvorsen A and Rosland J. Labour analgesia: A randomised, controlled trial comparing intravenous remifentanil and epidural analgesia with ropivacaine and fentanyl. Eur J Anaesthesiol 2012;29:129-36. 